\title{
La maîtrise des maladies infectieuses
}

\section{Un défi de santé publique, une ambition médico-scientifique}

RAPPORT SUR LA SCIENCE ET LA TECHNOLOGIE N 24

Animateurs : Gérard Orth et Philippe Sansonetti

ACADÉMIE DES SCIENCES

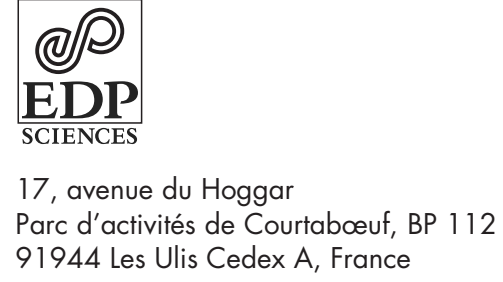


Rapports sur la science et la technologie

- Sciences et pays en développement. Afrique subsaharienne francophone RST n²1, 2006.

- La recherche spatiale française

RST n²2, 2006.

- L'épidémiologie humaine. Conditions de son développement en France, et rôle des mathématiques

RST n²3, 2006.

Imprimé en France

(C) 2006, EDP Sciences, 17, avenue du Hoggar, BP 112, Parc d'activités de Courtabœuf, 91944 Les Ulis Cedex A

Tous droits de traduction, d'adaptation et de reproduction par tous procédés réservés pour tous pays. Toute reproduction ou représentation intégrale ou partielle, par quelque procédé que ce soit, des pages publiées dans le présent ouvrage, faite sans l'autorisation de l'éditeur est illicite et constitue une contrefaçon. Seules sont autorisées, d'une part, les reproductions strictement réservées à I'usage privé du copiste et non destinées à une utilisation collective, et d'autre part, les courtes citations justifiées par le caractère scientifique ou d'information de l'œuvre dans laquelle elles sont incorporées (art. L. 122-4, L. 122-5 et L. 335-2 du Code de la propriété intellectuelle). Des photocopies payantes peuvent être réalisées avec l'accord de l'éditeur. S'adresser au : Centre français d'exploitation du droit de copie, 3, rue Hautefeuille, 75006 Paris. Tél. : 0143269535. 